\title{
'|||||||||||||||||||||||||||||||||||||||||||||||||||||||||||||||||||.
}

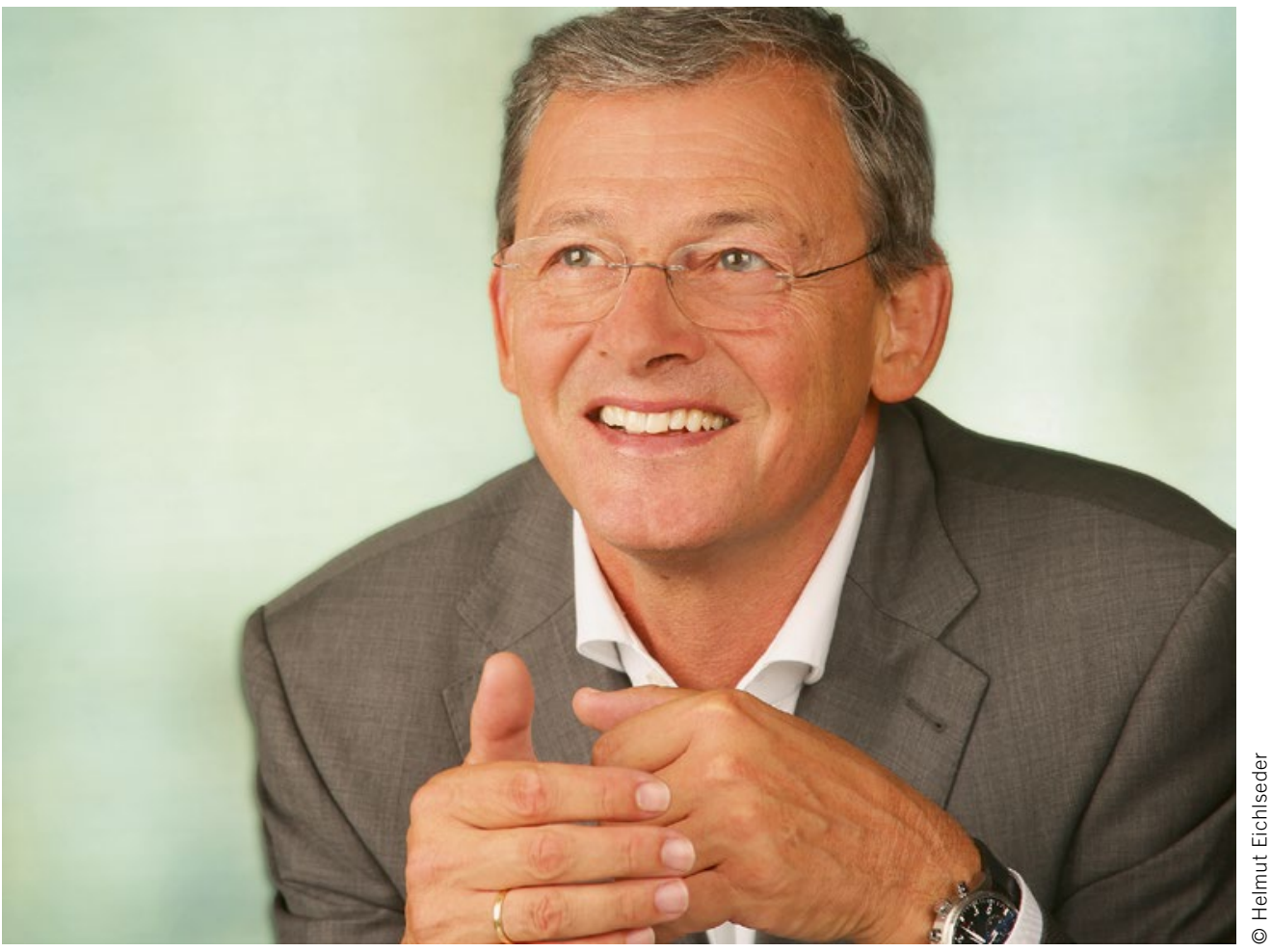

Univ. Prof. Dr. Helmut Eichlseder Head of the Institute of Internal Combustion Engines and Thermodynamics (IVT) at TU Graz

\section{Zero Impact - What Does It Really Mean?}

The term "Zero Impact Vehicle" (ZIV) has been widely used in recent times to describe the effect on the environment of future powertrains. A distinction is not always made between the climate relevance and pollution emissions of zero impact vehicles, but most people will agree that both are important. What is much less clear is the absolute level of pollution emissions and impacts on the climate that allow a vehicle to be described as a ZIV.

A number of possible approaches can be taken in order to achieve an exact definition of the emission level, disregarding for the moment the descriptions "no longer environmentally relevant" or "no significant impact on air quality." The spectrum ranges from "the well-to-wheel emissions are the same as a BEV" - which on the basis of German electricity generation are higher than those from one of the latest Euro 6 models - to "the $\mathrm{NO}_{2}$ concentration in the exhaust gas is below the air quality threshold." For me, a plausible definition is that the car's contribution to the emissions on busy streets is of the same order of magnitude as background values in rural areas with clean air, for example $\mathrm{NO}_{2}$ emissions of around $3.5 \mu \mathrm{g} / \mathrm{m}^{3}$. Many Euro 6 models already fall below these thresholds. The forthcoming Euro 7 legislation will require the entire fleet to comply with the zero impact definition above. When defining the limits and developing and using new emission reduction technologies, we should not be aiming at the maximum that is technically feasible, but instead focusing on the requirements of air quality.

The standards for the ZIV are much harder to meet in terms of climate relevance. Or they are provided that they are not based on the current, technically inaccurate tank-to-wheel approach with regard to $\mathrm{CO}_{2}$, but instead focus on the overall system (the Life Cycle Analysis or LCA). Even if only the energy supply is taken into consideration, zero impact vehicles are only possible from a climate perspective with the use of renewable energy.

Here there are three possible routes to take: electricity, hydrogen or renewable fuels with the appropriate energy converters or a combination of two or more of them in the vehicle. All three solutions have different advantages and disadvantages which make them suitable for different applications. A "universal solution" does not exist, contrary to widespread opinion.

In addition to the purely technical evaluation, other important criteria include affordability for broad sections of the population, the added value within Europe and the (backward) compatibility with the existing fleet and infrastructure. This is the only way that we can make the genuine reduction in our climate impact that is essential in the near future.

Combustion engines running on e-fuels, which include hydrogen, have the potential to meet the criteria for a zero impact powertrain convincingly in all the relevant areas. 